\title{
Content Type Name
}

National Cancer Institute

\section{Source}

National Cancer Institute. Content Type Name. NCI Thesaurus. Code C114549.

The text label assigned to a content type. 